\title{
Acesso posterior para implante de peso de ouro
}

\author{
Posterior approach to gold weight implant
}

\author{
Filipe José Pereira ${ }^{1}$ \\ Felipe Eing'2 \\ Francisco Azevedo Marquardt ${ }^{3}$ \\ Rodrigo Cavalheiro ${ }^{4}$ \\ Antonio Augusto Velasco eCruz ${ }^{5}$
}

\begin{tabular}{|l|}
\hline RESUMO \\
\hline Objetivo: Demonstrar uma técnica inovadora de implante de peso de ouro \\
via posterior e avaliar sua efetividade e possíveis complicações. Os \\
resultados serão comparados com a literatura existente sobre a técnica \\
via anterior, há muito tempo pouco modificada. Métodos: Foi realizado um \\
estudo prospectivo (seqüência de casos) com pacientes que apresenta- \\
vam, há mais de 6 meses, lagoftalmo paralítico, independentemente da \\
etiologia, atendidos no Departamento de Oculoplástica do Serviço de \\
Oftalmologia do Hospital Governador Celso Ramos - SC, entre o período \\
de fevereiro de 2006 a fevereiro de 2007 , com indicação do implante de \\
peso de ouro na pálpebra superior. A nova técnica via posterior foi \\
realizada por apenas dois cirurgiões. Resultados: Treze pacientes com \\
lagoftalmo paralítico, 9 homens e 4 mulheres com idade média de 53,07 \\
anos (variando de 17 a 73 ), foram submetidos ao implante de peso de ouro \\
pela técnica via posterior. O período de acompanhamento desses pa- \\
cientes foi de 2 meses a 1 ano, com média de 6,30 meses. Em 3 pacientes, \\
o peso implantado causou assimetria na distância margem-reflexo (DMR) \\
na posição primária do olhar - ptose de 2 mm em 2 pacientes e 4 mm em \\
1 paciente. Conclusões: Embora tal técnica venha exibindo um resultado \\
sistematicamente satisfatório, os autores acreditam que seja essencial o \\
acompanhamento dos pacientes por um tempo maior a fim de comprovar \\
a sustentabilidade de sua eficácia.
\end{tabular}

Descritores: Paralisia facial/complicações; Doenças palpebrais/etiologia; Doenças palpebrais/cirurgia; Ouro
Trabalho realizado no Departamento de Plástica Ocular do Hospital Governador Celso Ramos - Florianópolis - SC. Centro de Estudos de Oftalmologia Dr. Aramis Ritzmann Mendes.

${ }^{1}$ Pós-graduando, Chefe do Departamento de Plástica Ocular do Hospital Governador Celso Ramos - HGCR Florianópolis (SC) - Brasil.

${ }^{2}$ Residente do terceiro ano de Oftalmologia do HGCR Florianópolis (SC) - Brasil.

${ }^{3}$ Residente do segundo ano de Oftalmologia do HGCR Florianópolis (SC) - Brasil.

${ }^{4}$ Médico oftalmologista formado no Serviço de Oftalmologia HGCR - Florianópolis (SC) - Brasil.

${ }_{5}^{5}$ Professor Titular do Departamento de Oftalmologia, Otorrinolaringologia e Cirurgia de Cabeça e Pescoço da Faculdade de Medicina de Ribeirão Preto - Universidade de São Paulo - USP - Ribeirão Preto (SP) - Brasil.

Endereço para correspondência: Filipe José Pereira Rua Cristóvão Nunes Pires, 170 - Apto. 804 - Florianópolis (SC) CEP 88010-120

E-mail: filipejpereira@hotmail.com

Recebido para publicação em 03.08.2007

Última versão recebida em 25.05.2008

Aprovação em 11.06.2008

\section{INTRODUÇÃO}

A paralisia facial é uma doença de impacto por causar prejuízo funcional e estético. Dentre as causas de paralisia facial, as mais freqüentes são paralisia de Bell ou idiopática, tumores (neurinoma do nervo facial, neurinoma do nervo acústico, glômico, adenoma pleomórfico de parótida, carcinoma mucoepidermóide de parótida, melanoma temporal, sarcoma de articulação têmporo-mandibular, carcinoma de meato acústico externo, colesteatoma congênito, schwanoma), acidente vascular cerebral, pós-cirúrgico, infecção e trau$\mathrm{ma}^{(1)}$. Por sua vez, as manifestações clínicas mais comuns da paralisia facial periférica são lagoftalmo, diminuição da freqüência do piscar, ectrópio de pálpebra inferior com falha do mecanismo de bomba lacrimal, ptose do supercílio e queda ("sagging") do tecido periorbitário ${ }^{(2)}$.

Quanto ao lagoftalmo paralítico, ele consiste no fechamento palpebral incompleto originado pela perda da função do músculo orbicular, alterando a distribuição do filme lacrimal sobre a superfície ocular - o que poderá acarretar desde uma simples irritação até seqüelas visuais por perfuração de úlcera corneana $^{(3)}$. 
O tratamento inicial do lagoftalmo paralítico é clínico, realizado através de medicamentos lubrificantes, lentes de contato terapêuticas, câmara úmida e fechamento dos pontos lacrimais por meio de colocação de plugues, proporcionando conforto e proteção da córnea contra trauma e ressecamento ${ }^{(4)}$. Nos casos em que o tratamento conservador não fornece proteção corneana suficiente, é indicado o tratamento cirúrgico com o objetivo de restituir a dinâmica palpebral, através da aplicação das seguintes técnicas: cauterização ou oclusão cirúrgica de pontos lacrimais, blefarorrafia, tarsorrafia, cantoplastia, desinserção da aponeurose do levantador da pálpebra superior e implante de peso de ouro em pálpebra superior, sendo este último o mais comumente utilizado ${ }^{(4)}$.

O implante do peso de ouro para a correção do lagoftalmo proporciona a oclusão palpebral pela ação da gravidade $^{(5)}$. O motivo da escolha do ouro nessa técnica cirúrgica decorre das suas características físico-químicas, como a alta densidade, maleabilidade, pouca reatividade, coloração adequada para camuflagem abaixo da pele fina da pálpebra superior e fácil comercialização ${ }^{(6)}$. A popularidade desse tipo de implante aumentou gradativamente entre os cirurgiões oculoplásticos nos últimos 20 anos - o que resultou no surgimento de várias técnicas de implantação, como também em novas complicações ${ }^{(5)}$.

Há diversas técnicas cirúrgicas para implantação do peso de ouro, cuja seleção varia entre os pesos disponíveis comercialmente no Brasil: 0,8, 1,0,1,2 e 1,4 gramas. A cirurgia é feita ou por fixação externa do peso com cola ou adesivo (escolha terapêutica), ou por implante cirúrgico através de incisão no sulco palpebral, seguida de divulsão do músculo orbicular e fixação do peso, seja em placa tarsal, seja sob a aponeurose do levantador da pálpebra superior, seja atrás do septo orbitário ${ }^{(5,7-8)}$. Com o objetivo de diminuir as complicações pós-operatórias, alguns cirurgiões optam por recobrir o peso de ouro com fáscia temporal e pericárdio ${ }^{(9-10)}$.

As complicações atribuídas a esse procedimento cirúrgico incluem o lagoftalmo residual, a blefaroptose cobrindo o eixo visual, o entrópio, deformidades da pálpebra superior, protuberância do peso de ouro, reação inflamatória local, infecções, migração e extrusão do peso de ouro, sendo estes dois últimos os mais comuns e temíveis ${ }^{(11-12)}$.

Assim, com respeito ao lagoftalmo paralítico, o objetivo do presente estudo consiste em demonstrar uma técnica inovadora de implante de peso de ouro via posterior (ausente na literatura) e em avaliar sua efetividade e possíveis complicações. Os resultados serão comparados com a literatura existente sobre a técnica via anterior, há muito tempo pouco modificada.

\section{MÉTODOS}

Foi realizado um estudo prospectivo (seqüência de casos) em pacientes que apresentavam, há mais de seis meses, lagoftalmo paralítico (independentemente da etiologia) e, portanto, com indicação do implante de peso de ouro, atendidos no Departamento de Oculoplástica do Serviço de Oftalmologia do
Hospital Governador Celso Ramos (Santa Catarina) entre o período de fevereiro de 2006 a fevereiro de 2007.

Para escolha do peso ideal $(0,8,1,0,1,2$ ou 1,4 gramas), foram testados os pesos fixando-os com fita adesiva na pálpebra acometida e observando o fechamento palpebral e a DMR (distância margem-reflexo) superior resultante. Com isso, foi possível escolher o peso com melhor poder de correção do lagoftalmo e simetria com a pálpebra superior contralateral em posição primária do olhar. Vale salientar que todos os pacientes submetidos à implantação do peso de ouro com a técnica via posterior foram operados por apenas dois cirurgiões.

O sucesso do tratamento foi definido funcionalmente, levando-se em conta a eliminação da ceratopatia de exposição determinada pelo exame biomicroscópico, a preservação do eixo visual e a ausência de complicações no pós-operatório, tais como, migração, extrusão, inflamação, infecção e resultado cosmético ruim.

Por fim, realizou-se uma análise estatística descritiva dos resultados.

\section{Técnica cirúrgica}

A técnica cirúrgica utilizada compreende os seguintes passos, respectivamente:

- Anestesia local de pálpebra superior com lidocaína a $2 \%$ e com adrenalina a 1:200.000;

- Sutura marginal com seda 4-0 para estabilização da eversão palpebral com o afastador de Desmarres;

- Incisão vertical do tarso em sua porção lateral (via posterior) na largura do peso de ouro $(0,4 \mathrm{~mm})$, com lâmina de bisturi № 15 (Figura 1);

- Divulsão entre músculo orbicular pré-tarsal e tarso com tesoura Westcott na largura e no comprimento do peso de ouro (Figura 2);

- Inserção do peso de ouro (Figura 3);

- Sutura da abertura tarso-conjuntival em "U" invertido com vicryl 6.0 (Figura 4);

- Retirada da rédea sem necessidade de curativo.

\section{RESULTADOS}

Durante o período de um ano do estudo, 13 pacientes com lagoftalmo paralítico, 9 homens e 4 mulheres com idade média de 53,7 anos (variando de 17 a 73), foram submetidos ao implante de peso de ouro pela técnica nova técnica via posterior. Em 12 pacientes foram realizados concomitantemente outros procedimentos cirúrgicos: suspensão do supercílio, correção do ectrópio, correção da retração palpebral inferior e punctoplastia.

$\mathrm{O}$ peso implantado variou de 0,8 a $1,4 \mathrm{~g}$, conforme a distribuição abaixo:

- $0,8 \mathrm{~g}=2$ pacientes

- $1,0 \mathrm{~g}=5$ pacientes

- $1,2 \mathrm{~g}=4$ pacientes

- $1,4 \mathrm{~g}=2$ pacientes 


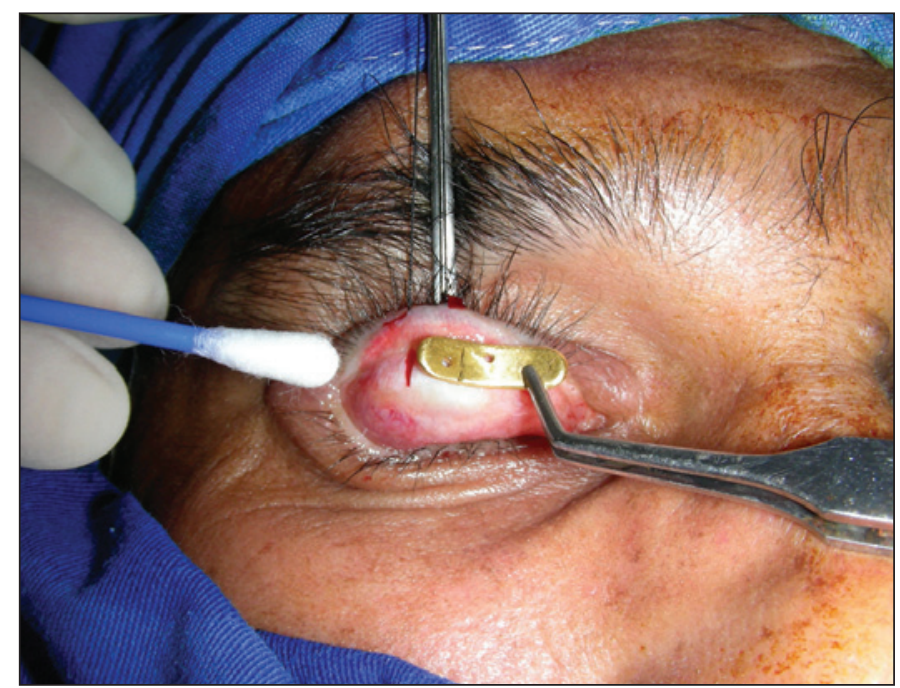

Figura 1 - Incisão vertical do tarso com lâmina de bisturi № 15 em sua porção lateral (via posterior) da largura do peso

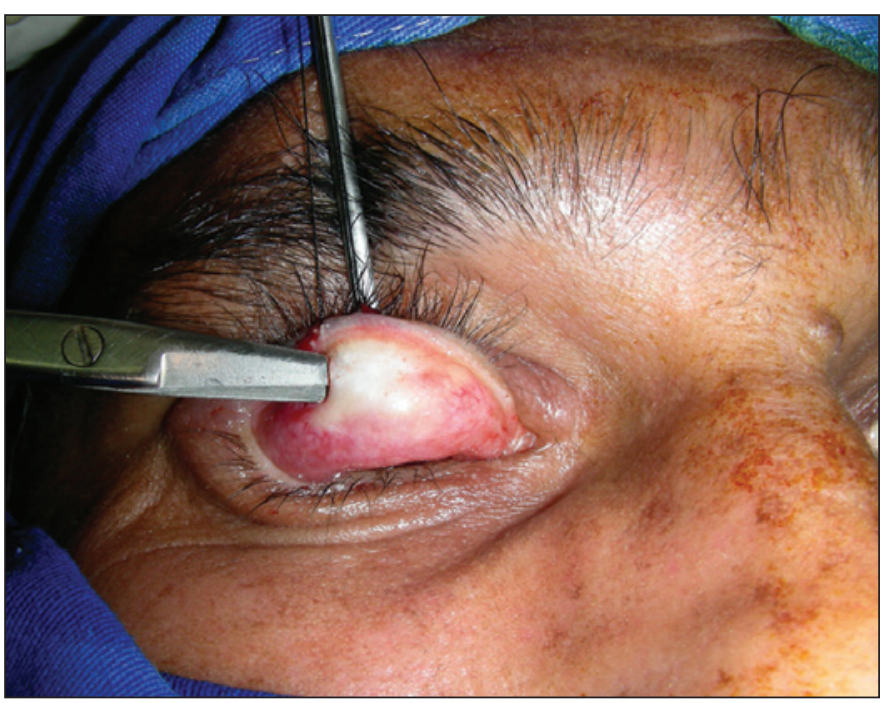

Figura 2 - Divulsão entre músculo orbicular pré-tarsal e tarso com tesoura Westcott na largura e comprimento do peso de ouro

O período de acompanhamento pós-operatório desses pacientes teve duração de 2 meses a 1 ano, com uma média de 6,3 meses. Todos os pacientes obtiveram melhora dos sintomas oculares e diminuição do uso de colírios lubrificantes, sendo que nenhum paciente ficou com lagoftalmo residual. Em apenas três pacientes o peso implantado causou assimetria na DMR na posição primária do olhar: ptose de $2 \mathrm{~mm}$ em dois deles e de $4 \mathrm{~mm}$ no outro.

É importante mencionar que não houve nenhum caso de infecção no pós-operatório, nem outras complicações durante o período de acompanhamento, como: entrópio, deformidade da pálpebra superior, migração ou extrusão.

A tabela 1 sumariza os dados dos 13 pacientes submetidos à implantação de peso de ouro.

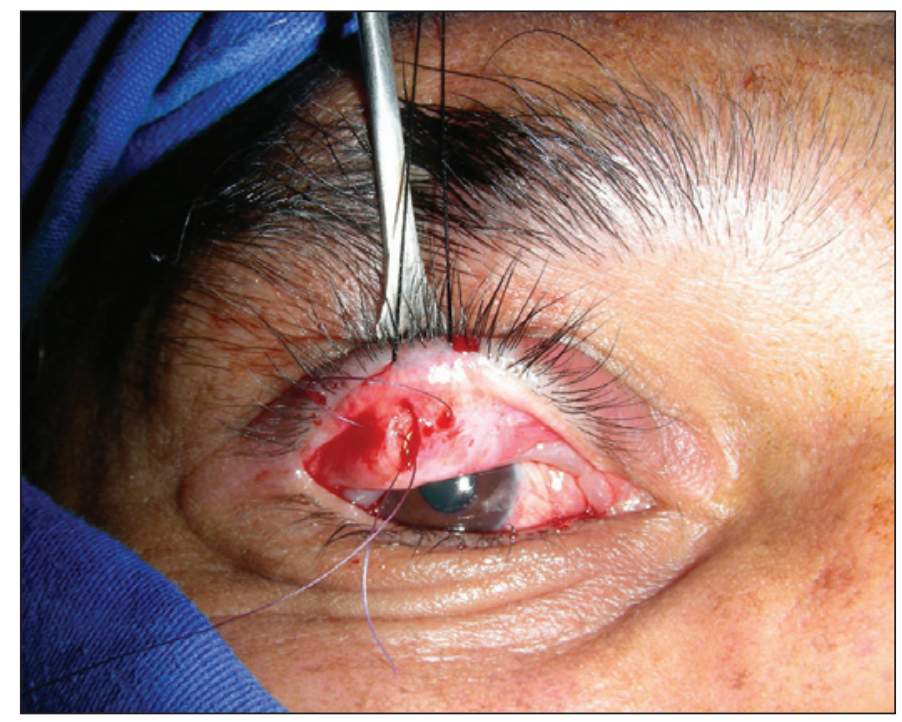

Figura 3 - Inserção do peso de ouro

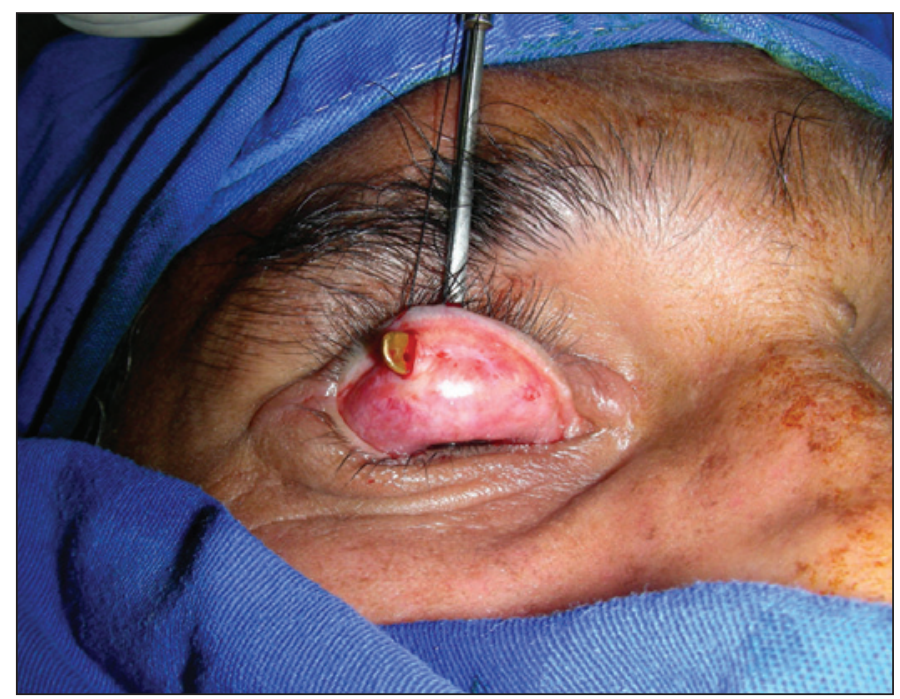

Figura 4 - Sutura da abertura tarso-conjuntival em "U" invertido com vicryl 6.0

\section{DISCUSSÃO}

Diversas técnicas cirúrgicas, com graus variáveis de sucesso e aceitação pelos pacientes, foram desenvolvidas para a reabilitação palpebral da paralisia facial, a fim de evitar complicações oculares que possam levar à cegueira.

Idealmente, qualquer método que seja adotado para a correção do lagoftalmo decorrente da paralisia facial deve alcançar as seguintes expectativas: proteção corneana adequada, ausência de distúrbios visuais, aceitação estética, baixo índice de complicações, simetria facial, possibilidade de reversão do procedimento nos casos de regressão da paralisia facial e facilidade técnica.

Dentre os procedimentos cirúrgicos existentes para o lagoftalmo, a implantação do peso de ouro passou a ser considera- 


\begin{tabular}{|c|c|c|c|c|c|}
\hline Paciente & Idade & Sexo & Causa da paralisia facial & $\begin{array}{c}\text { Procedimentos cirúrgicos } \\
\text { associados }\end{array}$ & $\begin{array}{c}\text { Tempo de } \\
\text { acompanhamento }\end{array}$ \\
\hline 1 & 54 & M & Trauma de mandíbula & Tarsal strip + spacer & 2 meses \\
\hline 2 & 41 & M & $\begin{array}{c}\text { Após cirurgia de } \\
\text { neurinoma do acústico }\end{array}$ & Spacer & 3 meses \\
\hline 3 & 42 & M & $\begin{array}{l}\text { Após cirurgia de tumor de } \\
\text { face (sem diagnóstico) }\end{array}$ & $\begin{array}{l}\text { Suspensão de supercílio } \\
+ \text { tarsal strip }+3 \text { snip }\end{array}$ & 10 meses \\
\hline 4 & 66 & M & Congênito & $\begin{array}{c}\text { Tarsal strip }+3 \text { snip }+ \\
\text { cantoplastia }\end{array}$ & 5 meses \\
\hline 5 & 63 & M & $\begin{array}{c}\text { Após acidente vascular } \\
\text { cerebral }\end{array}$ & Suspensão de supercílio & 5 meses \\
\hline 6 & 73 & $\mathrm{~F}$ & Paralisia de Bell & Tarsal strip & 4 meses \\
\hline 7 & 17 & M & $\begin{array}{c}\text { Após cirurgia de tumor } \\
\text { cerebral }\end{array}$ & - & 10 meses \\
\hline 8 & 58 & M & $\begin{array}{c}\text { Após cirurgia de tumor } \\
\text { cerebral }\end{array}$ & $\begin{array}{l}\text { Suspensão de supercílio } \\
+ \text { tarsal strip }+3 \text { snip }\end{array}$ & 10 meses \\
\hline 9 & 67 & $\mathrm{~F}$ & Paralisia de Bell & Tarsal strip & 9 meses \\
\hline 10 & 45 & M & Paralisia de Bell & $\begin{array}{l}\text { Suspensão de supercílio } \\
+ \text { tarsal strip + tarsorrafia }\end{array}$ & 11 meses \\
\hline 11 & 66 & M & $\begin{array}{c}\text { Após cirurgia de tumor } \\
\text { cerebral }\end{array}$ & $\begin{array}{c}\text { Tarsal strip }+ \text { tarsorrafia } \\
+3 \text { snip }\end{array}$ & 10 meses \\
\hline 12 & 53 & $\mathrm{~F}$ & Neurotoxoplasmose & - & 2 meses \\
\hline 13 & 45 & $\mathrm{~F}$ & $\begin{array}{c}\text { Após cirurgia de } \\
\text { neurinoma do acústico }\end{array}$ & Tarsal strip & 2 meses \\
\hline \multicolumn{6}{|c|}{$M=$ masculino; $F=$ feminino } \\
\hline
\end{tabular}

da por muitos cirurgiões como a mais eficaz para o tratamento dessa doença $a^{(2)}$. Tal dado motivou a realização deste trabalho, tendo em vista que as diversas técnicas de implantação, via anterior, descritas na literatura pouco se modificaram ao longo do tempo - todas com acesso transcutâneo, inicialmente sem fixação (suturas diretas no peso), mas, há muitos anos, já com suturas de fixação do peso no tarso, septo ou músculo levantador da pálpebra superior ${ }^{(5)}$.

Apesar da alta satisfação do paciente com o implante do peso de ouro, a incidência de complicações documentada na literatura encontra-se entre $0,5 \%$ e $61 \%$, constando a migração e a extrusão do peso de ouro como as principais ${ }^{(5)}$. Essa última é ainda, de todas as complicações possíveis, a mais temida e citada como a causa mais comum de falha no tratamento em até $15 \%$ dos $\operatorname{casos}^{(11-13)}$, sendo uma possível justificativa a diminuição do tônus da pele e do músculo orbicular ${ }^{(12)}$. Dentre os artigos revistos, somente Choi et al. citam uma média de 45,9 meses após a cirurgia para ocorrer a extrusão; porém, não relacionam esses casos de extrusão com períodos possíveis em que a mesma possa ocorrer ${ }^{(4)}$.

É importante ressaltar, ainda, que o implante do peso de ouro via transcutânea apresenta mais algumas desvantagens, como por exemplo, a demanda de um longo tempo cirúrgico pela necessidade de fixação do peso de ouro e sutura da pele, sangramento que exige o uso de cautério, e uma cicatriz cirúrgica.

Como todos os cirurgiões oculoplásticos almejam uma técnica cirúrgica fácil, de rápida execução e com bom resul- tado, os autores apresentam aqui uma técnica cirúrgica inovadora - via posterior - que tem revelado ser um excelente método para a correção do lagoftalmo paralítico, haja vista os bons resultados funcionais e estéticos obtidos em 13 pacientes em um ano de sua implementação. Ela é efetuada de forma rápida, tecnicamente fácil, sem a necessidade de sutura de fixação e retirada de pontos, além de rápida recuperação no pós-operatório e de dispensar o uso do cautério, possibilitando, assim, sua realização no leito, caso necessário.

A eficácia deste método em relação à extrusão ainda não é conclusiva, uma vez que a literatura não relata o período em que a mesma ocorre. Adicionalmente, os pacientes do presente estudo estão sendo acompanhados por no máximo 1 ano, período julgado insuficiente para tal avaliação.

Após a implantação do peso de ouro nos treze pacientes referidos, com tempo de acompanhamento variando de 2 meses a 1 ano, obteve-se como única complicação, conforme já relatado, assimetria na distância margem-reflexo superior de $2 \mathrm{~mm}$ em dois pacientes e de $4 \mathrm{~mm}$ em um paciente na posição primária do olhar, os quais não se queixaram do aspecto estético devido ao ótimo resultado funcional. Portanto, não houve nenhuma outra complicação como migração, infecção, ou outras já citadas como possíveis com a utilização do procedimento via anterior.

Sendo assim, o uso dessa nova técnica cirúrgica via posterior mostra-se eficaz pelo resultado consistente e satisfatório exibido até o momento. 


\section{CONCLUSÕES}

A nova técnica cirúrgica via posterior exposta no presente trabalho agrega as seguintes vantagens em relação ao procedimento tradicional: execução de forma rápida e tecnicamente fácil, sem necessidade de sutura de fixação e retirada de pontos, rápida recuperação no pós-operatório e dispensa do uso do cautério.

Contudo, embora tal técnica venha exibindo um resultado sistematicamente satisfatório, os autores acreditam que seja essencial o acompanhamento dos pacientes por um tempo maior a fim de comprovar a sustentabilidade de sua eficácia.

\section{ABSTRACT}

Purpose: To demonstrate an innovative technique of gold weight implantation through a posterior approach and evaluate its effectiveness and possible complications. The results will be compared to the other existing technique through anterior approach which has been unchanged for a long time, according to the literature. Methods: A prospective study (sequence of cases) was undertaken with patients who presented paralytic lagophthalmos for over 6 months. These patients presented many different etiologies and were assisted at the Department of Ophthalmology of Governador Celso Ramos Hospital-SC from February 2006 to February 2007. The implantation through posterior approach was performed by only 2 surgeons. Results: Thirteen patients with paralytic lagophthalmos, 9 men and 4 women, with an average age of 53.07 (range from 17 to 73 ) were submitted to a gold weight implantation through posterior approach. The follow-up period of those patients varied from 2 months to 1 year, with an average of 6.3 months. In 3 of the patients, the implanted weight caused asymmetry to the marginreflex distance (MRD) on the primary look position with ptosis of $2 \mathrm{~mm}$ in 2 patients and $4 \mathrm{~mm}$ in 1 patient. Conclusions: Although such technique has shown a satisfactory result, the authors believe that it is essential to extend the follow-up a little longer, so that the sustainability of its efficacy can be proved.

Keywords: Facial palsy/complications; Eyelid diseases/ etiology; Eyelid diseases/surgery; Gold

\section{REFERÊNCIAS}

1. Mavrikakis I, Beckingsale P, Lee E, Riaz Y, Brittain P. Changes in corneal topography with upper eyelid gold weight implants. Ophthal Plast Reconstr Surg. 2006;22(5):331-4.

2. Snyder MC, Johnson PJ, Moore GF, Ogren FP. Early versus late gold weight implantation for rehabilitation of the paralyzed eyelid. Laryngoscope. 2001; 111(12):2009-13.

3. Forno EA. Lagoftalmo paralítico. In: Matayoshi S, Forno EA, Moura EM. Manual de cirurgia plástica ocular. São Paulo: Roca; 2004. p. 79-86.

4. Choi HY, Hong SE, Lew JM. Long-term comparison of a newly designed gold implant with the conventional implant in facial nerve paralysis. Plast Reconstr Surg. 1999;104(6):1624-34. Comment in: Plast Reconstr Surg. 2000;106(3):735-6

5. Tower RN, Dailey RA. Gold weight implantation: a better way? Ophthal Plast Reconstr Surg. 2004;20(3):202-6.

6. Bhatti AF, Page K, Orlando A. Modification of the gold-weight implant for insertion into the upper eyelid in facial palsy. Ann Plast Surg. 2005;55(6): 689-92.

7. Hontanilla B. Weight measurement of upper eyelid gold implants for lagophthalmos in facial paralysis. Plast Reconstr Surg. 2001;108(6):1539-43. Comment in: Plast Reconstr Surg. 2004;114(6):1654-5; author reply 1655.

8. Shepler TR, Seiff SR. Use of isobutyl cyanoacrylate tissue adhesive to stabilize external eyelid weights in temporary treatment of facial palsies. Ophthal Plast Reconstr Surg. 2001;17(3):169-73.

9. Thomas DA, Khalifa YM. Temporalis fascia in the management of gold eyelid weight extrusion. Ophthal Plast Reconstr Surg. 2005;21(2):153-5.

10. Foster JA, Perry JD, Cahill KV, Holck DE, Kugler L. Processed human pericardium barrier for gold weight implantation. Ophthal Plast Reconstr Surg. 2004;20(2):107-9.

11. Seider N, Beiran I, Gdal-On M, Miller B. Posterior lamellar gold-weight extrusion. Ophthal Plast Reconstr Surg. 2003;19(5):407-8.

12. Nunes TP, Sardinha M, Pereira IC, Lunardelli P, Matayoshi S. Implante de peso de ouro: complicações precoces e tardias. Arq Bras Oftalmol. 2007;70 (4):599-602.

13. Pickford MA, Scamp T, Harrison DH. Morbidity after gold weight insertion into the upper eyelid in facial palsy. Br J Plast Surg. 1992;45(6):460-4. Comment in: Br J Plast Surg. 1993;46(4):343-4. Br J Plast Surg. 1993;46(4):343-4.

\section{Envie seu trabalho pelo site dos ABO}

www.aboonline.com.br 